Foley, E.K., et al., 2021, Jurassic Arc: Reconstructing the Lost World of eastern Gondwana: Geology, v. 49, https://doi.org/10.1130/G49328.1

\title{
Jurassic Arc: Reconstructing the lost world of eastern Gondwana
}

Elliot.K. Foley*, R.A. Henderson, E.M. Roberts, A.I.S. Kemp, C.N. Todd, E.M. Knutsen, C. Fisher, C.C. Wainman and Carl Spandler.

Department of Geosciences, James Cook University Townsville, Queensland 4811, Australia *E-mail contact: elliot.foley@my.jcu.edu.au

\section{SUPPLEMENTARY PAPERS}

Geology (2021)

\section{SUPPLEMENTARY PAPERS}

Supplementary file A. List of samples used for U-Pb geochronology in this study (PDF).

Supplementary file $\mathrm{B}$. U-Pb geochronological datasets (Excel file).

Supplementary file C. List of samples used for Lu-Hf analysis in this study (PDF).

Supplementary file D. Lu-Hf isotope analytical datasets (Excel file).

Supplementary file E. Analytical setup and procedures used (PDF).

Supplementary file F. Crustal addition rate calculations (PDF).

Supplementary file G. Supplementary Figure 1. Plotted $\varepsilon H f$ data with isotopic evolution curves (PDF). 
SUPPLEMENTARY DATA (\# corresponds to number of analyses accepted and used in this study)

\begin{tabular}{|c|c|c|c|c|c|c|c|c|c|c|}
\hline \multirow{2}{*}{$\begin{array}{c}\text { Sample } \\
\text { Name }\end{array}$} & \multirow{2}{*}{ \# } & \multicolumn{2}{|c|}{$\begin{array}{c}\text { Coordinates } \\
\text { (WGS84) }\end{array}$} & \multicolumn{2}{|c|}{ Sample Type } & \multirow{2}{*}{$\begin{array}{c}\text { Well } \\
\text { Name }\end{array}$} & \multirow{2}{*}{$\begin{array}{c}\text { Depth } \\
\text { (m) }\end{array}$} & \multirow{2}{*}{ Basin } & \multirow{2}{*}{ Formation } & \multirow{2}{*}{ Reference } \\
\hline & & Lat. & Long. & Core & $\begin{array}{c}\text { Outcr } \\
\text { op }\end{array}$ & & & & & \\
\hline PGF01 & 89 & $20.335259^{\circ} \mathrm{S}$ & $\begin{array}{c}144.469013^{\circ} \\
\mathrm{E}\end{array}$ & & $\mathrm{x}$ & - & - & Galilee Basin & $\begin{array}{l}\text { Porcupine Gorge } \\
\text { Formation }\end{array}$ & \\
\hline H7B59 & 68 & $20.950001^{\circ} \mathrm{S}$ & $\begin{array}{c}144.183329^{\circ} \\
\mathrm{E}\end{array}$ & $\mathrm{x}$ & & Hughenden 7 & 198.0 & Eromanga Basin & Hooray Sandstone & \\
\hline H7B 66 & 73 & $20.950001^{\circ} \mathrm{S}$ & $\begin{array}{c}144.183329^{\circ} \\
\mathrm{E}\end{array}$ & $\mathrm{x}$ & & Hughenden 7 & 230.0 & Eromanga Basin & Injune Creek Group & \\
\hline H7B55 & 86 & $20.950001^{\circ} \mathrm{S}$ & $\begin{array}{c}144.183329^{\circ} \\
\mathrm{E}\end{array}$ & $\mathrm{x}$ & & Hughenden 7 & 184.0 & Eromanga Basin & Gilbert River Formation & \\
\hline MMD-19-001 & 135 & $23.629325^{\circ} \mathrm{S}$ & $\begin{array}{c}50.370667^{\circ} \\
\mathrm{E}\end{array}$ & & $\mathrm{x}$ & - & - & Surat Basin & Razorback Beds & \\
\hline QMF7882 & 52 & $24.470004^{\circ} \mathrm{S}$ & $\begin{array}{c}150.699994^{\circ} \\
\mathrm{E}\end{array}$ & & $\mathrm{X}$ & - & - & Surat Basin & Evergreen Formation & $\begin{array}{l}\text { Todd et al. } \\
(2019)\end{array}$ \\
\hline QMF1659 & 69 & $24.626003^{\circ} \mathrm{S}$ & $\begin{array}{c}149.742999^{\circ} \\
\mathrm{E}\end{array}$ & & $\mathrm{x}$ & - & - & Surat Basin & Walloon Coal Measures & $\begin{array}{l}\text { Todd et al. } \\
\text { (2019) }\end{array}$ \\
\hline MV1 & 100 & $25.740955^{\circ} \mathrm{S}$ & $\begin{array}{c}152.468957^{\circ} \\
\mathrm{E}\end{array}$ & & $\mathrm{x}$ & - & - & Nambour Basin & Myrtle Creek Sandstone & \\
\hline MV2 & 125 & $25.750250^{\circ} \mathrm{S}$ & $\begin{array}{c}152.368119^{\circ} \\
\mathrm{E}\end{array}$ & & $\mathrm{x}$ & - & - & Nambour Basin & Myrtle Creek Sandstone & \\
\hline NM2 & 97 & $26.546349^{\circ} \mathrm{S}$ & $\begin{array}{c}153.102650^{\circ} \\
\mathrm{E}\end{array}$ & & $\mathrm{x}$ & - & - & Nambour Basin & Myrtle Creek Sandstone & \\
\hline NM3 & 114 & $26.380030^{\circ} \mathrm{S}$ & $\begin{array}{c}153.099495^{\circ} \\
\mathrm{E}\end{array}$ & & $\mathrm{x}$ & - & - & Nambour Basin & Myrtle Creek Sandstone & \\
\hline NM5 & 79 & $26.671758^{\circ} \mathrm{S}$ & $\begin{array}{c}153.109686^{\circ} \\
\mathrm{E}\end{array}$ & & $\mathrm{x}$ & - & - & Nambour Basin & Myrtle Creek Sandstone & \\
\hline DINO_TRACK & 119 & $20.143116^{\circ} \mathrm{S}$ & $\begin{array}{c}143.640990^{\circ} \\
\mathrm{E}\end{array}$ & & $\mathrm{x}$ & - & - & Eromanga Basin & Blantyre Sandstone & \\
\hline 081018-6 & 102 & $14.893964^{\circ} \mathrm{S}$ & $\begin{array}{c}143.974568^{\circ} \\
\mathrm{E}\end{array}$ & $\mathrm{x}$ & & Lakefield 1 & 389.5 & Laura Basin & Gilbert River Formation & \\
\hline $101018-2$ & 89 & $19.295442^{\circ} \mathrm{S}$ & $\begin{array}{c}140.381436^{\circ} \\
\mathrm{E}\end{array}$ & $\mathrm{x}$ & & Dobbyn 1 & 35.3 & $\begin{array}{l}\text { Carpentaria } \\
\text { Basin }\end{array}$ & Normanton Formation & \\
\hline $101018-4$ & 84 & $14.535470^{\circ} \mathrm{S}$ & $\begin{array}{c}143.862274^{\circ} \\
\mathrm{E}\end{array}$ & $\mathrm{x}$ & & Ebagoola 1 & 339.5 & Laura Basin & Gilbert River Formation & \\
\hline RP1-DZ-UGR & 112 & $15.656832^{\circ} \mathrm{S}$ & $\begin{array}{c}141.856686^{\circ} \\
\mathrm{E}\end{array}$ & $\mathrm{x}$ & & Rutland Plains 1 & 1005.5 & $\begin{array}{l}\text { Carpentaria } \\
\text { Basin }\end{array}$ & Gilbert River Formation & $\begin{array}{l}\text { Foley et al. } \\
\text { (2021) }\end{array}$ \\
\hline
\end{tabular}




\begin{tabular}{|c|c|c|c|c|c|c|c|c|c|c|}
\hline W1-DZ-UGR & 129 & $12.785066^{\circ} \mathrm{S}$ & $\begin{array}{c}142.404824^{\circ} \\
\mathrm{E}\end{array}$ & $\mathrm{X}$ & & Weipa 1 & 429.6 & $\begin{array}{c}\text { Carpentaria } \\
\text { Basin }\end{array}$ & Gilbert River Formation & $\begin{array}{l}\text { Foley et al. } \\
(2021)\end{array}$ \\
\hline 101018-34 & 91 & $17.715162^{\circ} \mathrm{S}$ & $\begin{array}{c}141.034461^{\circ} \\
\mathrm{E}\end{array}$ & $\mathrm{X}$ & & Normanton 1 & 94.8 & $\begin{array}{c}\text { Carpentaria } \\
\text { Basin }\end{array}$ & Normanton Formation & \\
\hline 081018-12 & 96 & $14.737267^{\circ} \mathrm{S}$ & $\begin{array}{c}143.919729^{\circ} \\
\mathrm{E}\end{array}$ & $\mathrm{X}$ & & Breeza Plains 1 & 495.0 & Laura Basin & Dalrymple Sandstone & \\
\hline $\begin{array}{l}\text { Top Middle } \\
\text { Loth }\end{array}$ & 103 & $19.742728^{\circ} \mathrm{S}$ & $\begin{array}{c}143.630618^{\circ} \\
\mathrm{E}\end{array}$ & & $\mathrm{X}$ & - & - & $\begin{array}{c}\text { Carpentaria } \\
\text { Basin }\end{array}$ & Loth Formation & \\
\hline McKinlay-02 & 63 & $21.592507^{\circ} \mathrm{S}$ & $\begin{array}{c}142.259717^{\circ} \\
\mathrm{E}\end{array}$ & $\mathrm{X}$ & & McKinlay-1 & 9.3 & Eromanga Basin & Winton Formation & $\begin{array}{l}\text { Tucker et al. } \\
\text { (2016) }\end{array}$ \\
\hline McKinlay-16 & 63 & $21.592507^{\circ} \mathrm{S}$ & $\begin{array}{c}142.259717^{\circ} \\
\mathrm{E}\end{array}$ & $\mathrm{X}$ & & McKinlay-1 & 54.0 & Eromanga Basin & Winton Formation & $\begin{array}{l}\text { Tucker et al. } \\
\text { (2016) }\end{array}$ \\
\hline HadesHill & 40 & $23.016288^{\circ} \mathrm{S}$ & $\begin{array}{c}142.411865^{\circ} \\
\mathrm{E}\end{array}$ & & $\mathrm{x}$ & - & - & Eromanga Basin & Winton Formation & $\begin{array}{l}\text { Tucker et al. } \\
\text { (2016) }\end{array}$ \\
\hline ERU & 73 & $23.016288^{\circ} \mathrm{S}$ & $\begin{array}{c}142.411865^{\circ} \\
\mathrm{E}\end{array}$ & & $\mathrm{x}$ & - & - & Eromanga Basin & Winton Formation & $\begin{array}{l}\text { Tucker et al. } \\
\text { (2016) }\end{array}$ \\
\hline BBD4 & 81 & $22.514825^{\circ} \mathrm{S}$ & $\begin{array}{c}143.038857^{\circ} \\
\mathrm{E}\end{array}$ & & $\mathrm{X}$ & - & - & Eromanga Basin & Winton Formation & $\begin{array}{l}\text { Tucker et al. } \\
\text { (2016) }\end{array}$ \\
\hline Blackall-02 & 61 & $24.162590^{\circ} \mathrm{S}$ & $\begin{array}{c}144.223606^{\circ} \\
\mathrm{E}\end{array}$ & $\mathrm{X}$ & & Blackall 2 & 27.0 & Eromanga Basin & Winton Formation & $\begin{array}{l}\text { Tucker et al. } \\
\text { (2016) }\end{array}$ \\
\hline ERO1 & 92 & $26.614710^{\circ} \mathrm{S}$ & $\begin{array}{c}143.880111^{\circ} \\
\mathrm{E}\end{array}$ & $\mathrm{X}$ & & Eromanga 1 & 20.0 & Eromanga Basin & Winton Formation & $\begin{array}{l}\text { Tucker et al. } \\
\text { (2016) }\end{array}$ \\
\hline ISIS & 60 & $24.259307^{\circ} \mathrm{S}$ & $\begin{array}{c}144.441483^{\circ} \\
\mathrm{E}\end{array}$ & & $\mathrm{X}$ & - & - & Eromanga Basin & Winton Formation & $\begin{array}{l}\text { Tucker et al. } \\
\text { (2016) }\end{array}$ \\
\hline Longmac1 & 64 & $23.111931^{\circ} \mathrm{S}$ & $\begin{array}{c}144.599555^{\circ} \\
\mathrm{E}\end{array}$ & $\mathrm{X}$ & & Longreach 1 & 27.0 & Eromanga Basin & Mackunda Formation & $\begin{array}{l}\text { Tucker et al. } \\
\text { (2016) }\end{array}$ \\
\hline Maneroo-331 & 82 & $23.385865^{\circ} \mathrm{S}$ & $\begin{array}{c}144.467891^{\circ} \\
\mathrm{E}\end{array}$ & $\mathrm{X}$ & & Maneroo 1 & 343.0 & Eromanga Basin & Mackunda Formation & $\begin{array}{l}\text { Tucker et al. } \\
\text { (2016) }\end{array}$ \\
\hline MV6 & 85 & $25.925457^{\circ} \mathrm{S}$ & $\begin{array}{c}152.432065^{\circ} \\
\mathrm{E}\end{array}$ & & $\mathrm{x}$ & - & - & Nambour Basin & Tiaro Coal Measures & \\
\hline MV4 & 120 & $25.556158^{\circ} \mathrm{S}$ & $\begin{array}{c}152.460068^{\circ} \\
\mathrm{E}\end{array}$ & & $\mathrm{X}$ & - & - & Nambour Basin & Tiaro Coal Measures & \\
\hline MV15 & 5 & $25.551132^{\circ} \mathrm{S}$ & $\begin{array}{c}152.509764^{\circ} \\
\mathrm{E}\end{array}$ & & $\mathrm{X}$ & - & - & $\begin{array}{l}\text { Maryborough } \\
\text { Basin }\end{array}$ & Grahams Creek Formation & \\
\hline MV16 & 77 & $25.551491^{\circ} \mathrm{S}$ & $\begin{array}{c}152.509175^{\circ} \\
\mathrm{E}\end{array}$ & & $\mathrm{X}$ & - & - & $\begin{array}{l}\text { Maryborough } \\
\text { Basin }\end{array}$ & Grahams Creek Formation & \\
\hline MV17 & 59 & $25.559521^{\circ} \mathrm{S}$ & $\begin{array}{c}152.598749^{\circ} \\
\mathrm{E}\end{array}$ & & $\mathrm{X}$ & - & - & $\begin{array}{l}\text { Maryborough } \\
\text { Basin }\end{array}$ & Grahams Creek Formation & \\
\hline 2550367 & 55 & $28.044004^{\circ} \mathrm{S}$ & $\begin{array}{c}141.961701^{\circ} \\
\mathrm{E}\end{array}$ & $\mathrm{X}$ & & Zeus 7 & 1667.5 & Eromanga Basin & Birkhead Formation & $\begin{array}{l}\text { Wainman et al. } \\
\text { (2018) }\end{array}$ \\
\hline 2550364 & 57 & $28.044004^{\circ} \mathrm{S}$ & $\begin{array}{c}141.961701^{\circ} \\
\mathrm{E}\end{array}$ & $\mathrm{X}$ & & Zeus 7 & 1659.3 & Eromanga Basin & Birkhead Formation & $\begin{array}{c}\text { Wainman et al. } \\
\text { (2018) }\end{array}$ \\
\hline $\begin{array}{c}\text { Base } \\
\text { Hampstead }\end{array}$ & 102 & $19.885314^{\circ} \mathrm{S}$ & $\begin{array}{c}143.602908^{\circ} \\
\mathrm{E}\end{array}$ & & $\mathrm{X}$ & - & - & $\begin{array}{c}\text { Carpentaria } \\
\text { Basin }\end{array}$ & Hampstead Sandstone & $\begin{array}{l}\text { Foley et al. } \\
(2020)\end{array}$ \\
\hline $\begin{array}{l}\text { Base Upper } \\
\text { Loth }\end{array}$ & 114 & $19.742728^{\circ} \mathrm{S}$ & $\begin{array}{c}143.630618^{\circ} \\
\mathrm{E}\end{array}$ & & $\mathrm{X}$ & - & - & $\begin{array}{l}\text { Carpentaria } \\
\text { Basin }\end{array}$ & Loth Formation & $\begin{array}{l}\text { Foley et al. } \\
\text { (2020) }\end{array}$ \\
\hline Base Yappar & 113 & $19.845448^{\circ} \mathrm{S}$ & $\begin{array}{c}143.646430^{\circ} \\
\mathrm{E}\end{array}$ & & $\mathrm{X}$ & - & - & $\begin{array}{c}\text { Carpentaria } \\
\text { Basin }\end{array}$ & Gilbert River Formation & $\begin{array}{l}\text { Foley et al. } \\
\quad(2020)\end{array}$ \\
\hline
\end{tabular}




\begin{tabular}{|c|c|c|c|c|c|c|c|c|c|c|}
\hline $\begin{array}{l}\text { Base Lower } \\
\text { Loth }\end{array}$ & 100 & $19.857875^{\circ} \mathrm{S}$ & $\begin{array}{c}143.643210^{\circ} \\
\mathrm{E}\end{array}$ & & $\mathrm{X}$ & - & - & $\begin{array}{c}\text { Carpentaria } \\
\text { Basin }\end{array}$ & Loth Formation & $\begin{array}{l}\text { Foley et al. } \\
(2020)\end{array}$ \\
\hline $\begin{array}{l}\text { Base Middle } \\
\text { Loth }\end{array}$ & 72 & $19.742553^{\circ} \mathrm{S}$ & $\begin{array}{c}143.622213^{\circ} \\
\mathrm{E}\end{array}$ & & $\mathrm{X}$ & - & - & $\begin{array}{c}\text { Carpentaria } \\
\text { Basin }\end{array}$ & Loth Formation & $\begin{array}{l}\text { Foley et al. } \\
(2020)\end{array}$ \\
\hline D1-DZ-GR & 80 & $19.295442^{\circ} \mathrm{S}$ & $\begin{array}{c}140.381436^{\circ} \\
\mathrm{E}\end{array}$ & $\mathrm{X}$ & & Dobbyn 1 & 589.5 & $\begin{array}{c}\text { Carpentaria } \\
\text { Basin }\end{array}$ & Gilbert River Formation & $\begin{array}{l}\text { Foley et al. } \\
\quad(2021)\end{array}$ \\
\hline 031019-3 & 96 & $15.656832^{\circ} \mathrm{S}$ & $\begin{array}{c}141.856686^{\circ} \\
\mathrm{E}\end{array}$ & $\mathrm{X}$ & & Rutland Plains 1 & 1088.0 & $\begin{array}{c}\text { Carpentaria } \\
\text { Basin }\end{array}$ & Gilbert River Formation & $\begin{array}{l}\text { Foley et al. } \\
\text { (2021) }\end{array}$ \\
\hline 031019-6 & 118 & $12.785066^{\circ} \mathrm{S}$ & $\begin{array}{c}142.404824^{\circ} \\
\mathrm{E}\end{array}$ & $\mathrm{X}$ & & Weipa 1 & 519.9 & $\begin{array}{c}\text { Carpentaria } \\
\text { Basin }\end{array}$ & Gilbert River Formation & $\begin{array}{l}\text { Foley et al. } \\
(2021)\end{array}$ \\
\hline 031019-7 & 99 & $12.785066^{\circ} \mathrm{S}$ & $\begin{array}{c}142.404824^{\circ} \\
\mathrm{E}\end{array}$ & $\mathrm{X}$ & & Weipa 1 & 568.6 & $\begin{array}{c}\text { Carpentaria } \\
\text { Basin }\end{array}$ & Garraway Sandstone & $\begin{array}{l}\text { Foley et al. } \\
(2021)\end{array}$ \\
\hline 031019-13 & 57 & $19.295442^{\circ} \mathrm{S}$ & $\begin{array}{c}140.381436^{\circ} \\
\mathrm{E}\end{array}$ & $\mathrm{X}$ & & Dobbyn 1 & 44.7 & $\begin{array}{c}\text { Carpentaria } \\
\text { Basin }\end{array}$ & Normanton Formation & \\
\hline 041019-1 & 84 & $15.656832^{\circ} \mathrm{S}$ & $\begin{array}{c}141.856686^{\circ} \\
\mathrm{E}\end{array}$ & $\mathrm{X}$ & & Rutland Plains 1 & 351.1 & $\begin{array}{c}\text { Carpentaria } \\
\text { Basin }\end{array}$ & Normanton Formation & \\
\hline 041019-4 & 88 & $15.656832^{\circ} \mathrm{S}$ & $\begin{array}{c}141.856686^{\circ} \\
\mathrm{E}\end{array}$ & $\mathrm{X}$ & & Rutland Plains 1 & 270.4 & $\begin{array}{c}\text { Carpentaria } \\
\text { Basin }\end{array}$ & Normanton Formation & \\
\hline 041019-6 & 100 & $19.295442^{\circ} \mathrm{S}$ & $\begin{array}{c}140.381436^{\circ} \\
\mathrm{E}\end{array}$ & $\mathrm{X}$ & & Dobbyn 1 & 580.7 & $\begin{array}{c}\text { Carpentaria } \\
\text { Basin }\end{array}$ & Gilbert River Formation & $\begin{array}{l}\text { Foley et al. } \\
\quad(2021)\end{array}$ \\
\hline 041019-8 & 99 & $19.295442^{\circ} \mathrm{S}$ & $\begin{array}{c}140.381436^{\circ} \\
\mathrm{E}\end{array}$ & $\mathrm{X}$ & & Dobbyn 1 & 559.1 & $\begin{array}{c}\text { Carpentaria } \\
\text { Basin }\end{array}$ & Gilbert River Formation & $\begin{array}{l}\text { Foley et al. } \\
\quad(2021)\end{array}$ \\
\hline 041019-9 & 92 & $19.295442^{\circ} \mathrm{S}$ & $\begin{array}{c}140.381436^{\circ} \\
\mathrm{E}\end{array}$ & $\mathrm{X}$ & & Dobbyn 1 & 550.8 & $\begin{array}{c}\text { Carpentaria } \\
\text { Basin }\end{array}$ & Gilbert River Formation & $\begin{array}{l}\text { Foley et al. } \\
\quad(2021)\end{array}$ \\
\hline 081018-2 & 56 & $16.485452^{\circ} \mathrm{S}$ & $\begin{array}{c}139.520874^{\circ} \\
\mathrm{E}\end{array}$ & $\mathrm{x}$ & & Mornington 1 & 139.6 & $\begin{array}{c}\text { Carpentaria } \\
\text { Basin }\end{array}$ & Normanton Formation & \\
\hline 081018-1 & 85 & $14.893964^{\circ} \mathrm{S}$ & $\begin{array}{c}143.974568^{\circ} \\
\mathrm{E}\end{array}$ & $\mathrm{X}$ & & Lakefield 1 & 566.9 & Laura Basin & Dalrymple Sandstone & \\
\hline 081019-3 & 27 & $17.715162^{\circ} \mathrm{S}$ & $\begin{array}{c}141.034461^{\circ} \\
\mathrm{E}\end{array}$ & $\mathrm{X}$ & & Normanton 1 & 630.5 & $\begin{array}{c}\text { Carpentaria } \\
\text { Basin }\end{array}$ & Gilbert River Formation & \\
\hline 081019-5 & 48 & $17.715162^{\circ} \mathrm{S}$ & $\begin{array}{c}141.034461^{\circ} \\
\mathrm{E}\end{array}$ & $\mathrm{X}$ & & Normanton 1 & 606.5 & $\begin{array}{c}\text { Carpentaria } \\
\text { Basin }\end{array}$ & Gilbert River Formation & \\
\hline 091019-1 & 8 & $17.715162^{\circ} \mathrm{S}$ & $\begin{array}{c}141.034461^{\circ} \\
\mathrm{E}\end{array}$ & $\mathrm{x}$ & & Normanton 1 & 114.3 & $\begin{array}{c}\text { Carpentaria } \\
\text { Basin }\end{array}$ & Normanton Formation & \\
\hline 091019-3 & 91 & $17.715162^{\circ} \mathrm{S}$ & $\begin{array}{c}141.034461^{\circ} \\
\mathrm{E}\end{array}$ & $\mathrm{X}$ & & Normanton 1 & 22.2 & $\begin{array}{c}\text { Carpentaria } \\
\text { Basin }\end{array}$ & Normanton Formation & \\
\hline 101018-14 & 91 & $14.535470^{\circ} \mathrm{S}$ & $\begin{array}{c}143.862274^{\circ} \\
\mathrm{E}\end{array}$ & $\mathrm{X}$ & & Ebagoola 1 & 896.2 & Laura Basin & Dalrymple Sandstone & \\
\hline $101018-27$ & 64 & $15.656832^{\circ} \mathrm{S}$ & $\begin{array}{c}141.856686^{\circ} \\
\mathrm{E}\end{array}$ & $\mathrm{X}$ & & Rutland Plains 1 & 1078.1 & $\begin{array}{c}\text { Carpentaria } \\
\text { Basin }\end{array}$ & Gilbert River Formation & $\begin{array}{l}\text { Foley et al. } \\
\quad(2021)\end{array}$ \\
\hline $101018-36$ & 68 & $14.634593^{\circ} \mathrm{S}$ & $\begin{array}{c}143.876096^{\circ} \\
\mathrm{E}\end{array}$ & $\mathrm{X}$ & & Marina 1 & 187.5 & Laura Basin & Rolling Downs Group & \\
\hline 121018-1 & 44 & $12.785066^{\circ} \mathrm{S}$ & $\begin{array}{c}142.404824^{\circ} \\
\mathrm{E}\end{array}$ & $\mathrm{X}$ & & Weipa 1 & 581.4 & $\begin{array}{c}\text { Carpentaria } \\
\text { Basin }\end{array}$ & Garraway Sandstone & $\begin{array}{l}\text { Foley et al. } \\
\quad(2021)\end{array}$ \\
\hline $121018-7$ & 131 & $12.785066^{\circ} \mathrm{S}$ & $\begin{array}{c}142.404824^{\circ} \\
\mathrm{E}\end{array}$ & $\mathrm{X}$ & & Weipa 1 & 392.3 & $\begin{array}{c}\text { Carpentaria } \\
\text { Basin }\end{array}$ & Rolling Downs Group & $\begin{array}{l}\text { Foley et al. } \\
\text { (2021) }\end{array}$ \\
\hline Top Hampstead & 100 & $19.857875^{\circ} \mathrm{S}$ & $\begin{array}{c}143.643210^{\circ} \\
\mathrm{E}\end{array}$ & & $\mathrm{X}$ & - & - & $\begin{array}{c}\text { Carpentaria } \\
\text { Basin }\end{array}$ & Hampstead Sandstone & $\begin{array}{l}\text { Foley et al. } \\
(2020)\end{array}$ \\
\hline
\end{tabular}




\begin{tabular}{|c|c|c|c|c|c|c|c|c|c|c|}
\hline Top Upper Loth & 139 & $19.845448^{\circ} \mathrm{S}$ & $\begin{array}{c}143.646430^{\circ} \\
\mathrm{E}\end{array}$ & & $\mathrm{X}$ & - & - & $\begin{array}{l}\text { Carpentaria } \\
\text { Basin }\end{array}$ & Loth Formation & $\begin{array}{l}\text { Foley et al. } \\
(2020)\end{array}$ \\
\hline $\begin{array}{l}\text { Top Lower } \\
\text { Loth }\end{array}$ & 128 & $19.742553^{\circ} \mathrm{S}$ & $\begin{array}{c}143.622213^{\circ} \\
\mathrm{E}\end{array}$ & & $\mathrm{X}$ & - & - & $\begin{array}{l}\text { Carpentaria } \\
\text { Basin }\end{array}$ & Loth Formation & $\begin{array}{c}\text { Foley et al. } \\
\text { (2020) }\end{array}$ \\
\hline W1-DZ-LGS & 90 & $12.785066^{\circ} \mathrm{S}$ & $\begin{array}{c}142.404824^{\circ} \\
\mathrm{E}\end{array}$ & $\mathrm{X}$ & & Weipa 1 & 573.6 & $\begin{array}{l}\text { Carpentaria } \\
\text { Basin }\end{array}$ & Garraway Sandstone & $\begin{array}{l}\text { Foley et al. } \\
\text { (2021) }\end{array}$ \\
\hline BGR & 94 & $20.403973^{\circ} \mathrm{S}$ & $\begin{array}{c}144.437436^{\circ} \\
\mathrm{E}\end{array}$ & & $\mathrm{X}$ & - & - & Eromanga Basin & Blantyre Sandstone & \\
\hline BSS-2004 & 87 & $20.374497^{\circ} \mathrm{S}$ & $\begin{array}{c}144.454432^{\circ} \\
\mathrm{E}\end{array}$ & & $\mathrm{X}$ & - & - & Eromanga Basin & Blantyre Sandstone & \\
\hline $\begin{array}{c}\text { Dalrymple } \\
\text { Hons }\end{array}$ & 117 & $15.288330^{\circ} \mathrm{S}$ & $\begin{array}{c}145.215559^{\circ} \\
\mathrm{E}\end{array}$ & & $\mathrm{X}$ & - & - & Laura Basin & Dalrymple Sandstone & \\
\hline GRF01 & 79 & $20.408712^{\circ} \mathrm{S}$ & $\begin{array}{c}144.436754^{\circ} \\
\mathrm{E}\end{array}$ & & $\mathrm{X}$ & - & - & Eromanga Basin & Blantyre Sandstone & \\
\hline H5B5 & 52 & $20.633335^{\circ} \mathrm{S}$ & $\begin{array}{c}144.399996^{\circ} \\
\mathrm{E}\end{array}$ & $\mathrm{X}$ & & Hughenden 5 & 53.5 & Eromanga Basin & Blantyre Sandstone & \\
\hline H5B21 & 83 & $20.633335^{\circ} \mathrm{S}$ & $\begin{array}{c}144.399996^{\circ} \\
E\end{array}$ & $\mathrm{X}$ & & Hughenden 5 & 105.0 & Eromanga Basin & Blantyre Sandstone & \\
\hline H6B8 & 90 & $20.346885^{\circ} \mathrm{S}$ & $\begin{array}{c}144.53219^{\circ} \\
\mathrm{E}\end{array}$ & $\mathrm{X}$ & & Hughenden 6 & 41.0 & Eromanga Basin & Blantyre Sandstone & \\
\hline H6B26 & 68 & $20.346885^{\circ} \mathrm{S}$ & $\begin{array}{c}144.53219^{\circ} \\
\mathrm{E}\end{array}$ & $\mathrm{X}$ & & Hughenden 6 & 120.0 & Galilee Basin & Warang Sandstone & \\
\hline H6B38 & 22 & $20.346885^{\circ} \mathrm{S}$ & $\begin{array}{c}144.53219^{\circ} \\
\mathrm{E}\end{array}$ & $\mathrm{X}$ & & Hughenden 6 & 169.0 & Galilee Basin & Warang Sandstone & \\
\hline H6B47 & 92 & $20.346885^{\circ} \mathrm{S}$ & $\begin{array}{c}144.53219^{\circ} \\
\mathrm{E}\end{array}$ & $\mathrm{X}$ & & Hughenden 6 & 199.0 & Galilee Basin & $\begin{array}{l}\text { Porcupine Gorge } \\
\text { Formation }\end{array}$ & \\
\hline H7B101 & 93 & $20.950001^{\circ} \mathrm{S}$ & $\begin{array}{c}144.183329^{\circ} \\
\mathrm{E}\end{array}$ & $\mathrm{X}$ & & Hughenden 7 & 341.0 & Eromanga Basin & Hutton Sandstone & \\
\hline Wall 1 & 77 & $20.408701^{\circ} \mathrm{S}$ & $\begin{array}{c}144.436611^{\circ} \\
\mathrm{E}\end{array}$ & & $\mathrm{X}$ & - & - & Eromanga Basin & Gilbert River Formation & \\
\hline WSS-Top & 81 & $20.351433^{\circ} \mathrm{S}$ & $\begin{array}{c}144.466663^{\circ} \\
\mathrm{E}\end{array}$ & & $\mathrm{X}$ & - & - & Galilee Basin & Warang Sandstone & \\
\hline WSS-0409 & 93 & $20.335468^{\circ} \mathrm{S}$ & $\begin{array}{c}144.469125^{\circ} \\
\mathrm{E}\end{array}$ & & $\mathrm{X}$ & - & - & Galilee Basin & Warang Sandstone & \\
\hline WSS-2908 & 94 & $20.335350^{\circ} \mathrm{S}$ & $\begin{array}{c}144.469088^{\circ} \\
\mathrm{E}\end{array}$ & & $X$ & - & - & Galilee Basin & Warang Sandstone & \\
\hline
\end{tabular}

\section{References}

Foley, E.K., Baty, M., Knusten, E.M., Lignum, J.S., and Roberts, E.M., 2020, Jurassic-Early Cretaceous paleogeography and paleoenvironments of the north-eastern margin of Gondwana: Insights from the Carpentaria Basin, Australia: Gondwana Research, v. 88, p. 126-149,

doi.org/10.1016/j.gr.2020.07.003. 
Foley, E. K., Roberts, E. M., Knutsen, E. M., and Hannaford, C., 2021, Cryptic Middle to Late Jurassic marine incursions into northeastern Gondwana: An integrated sedimentological, ichnological and geochronological approach: Palaeogeography, Palaeoclimatology, Palaeoecology, v. 569, 110330, doi.org/10.1016/j.palaeo.2021.110330

Todd, C.N., Roberts, E.M., Knutsen, E.M., Rozefelds, A.C., Huang, H.Q., and Spandler, C., 2019, Refined age and geological context of two of Australia's most important Jurassic vertebrate taxa (Rhoetosaurus brownie and Siderops kehli), Queensland: Gondwana Research, v. 76, p. 1925, doi.org/10.1016/j.gr.2019.05.008

Tucker, R.T., Roberts, E.M., Henderson, R.A., and Kemp, A.I.S., 2016, Large igneous province or long-lived magmatic arc along the eastern margin of Australia during the Cretaceous? Insights from the sedimentary record: Bulletin of the Geological Society of America, v. 128, p. 14611480, doi:10.1130/B31337.1.10.1130

Wainman, C. C., McCabe, P. J., and Crowley, J. L., 2018, Solving a tuff problem: Defining a chronostratigraphic framework for Middle to Upper Jurassic nonmarine strata in eastern Australia using uranium-lead chemical abrasion-thermal ionization mass spectrometry zircon dates: AAPG Bulletin, v. 102, p. 1141-1168, doi.org/10.1306/07261717140 